\title{
CONVERGENCE OF A KÄHLER-RICCI FLOW
}

\author{
NATASA Sesum
}

\begin{abstract}
In this paper we prove that for a given Kähler-Ricci flow with uniformly bounded Ricci curvatures in an arbitrary dimension, for every sequence of times $t_{i}$ converging to infinity, there exists a subsequence such that $\left(M, g\left(t_{i}+t\right)\right) \rightarrow$ $(Y, \bar{g}(t))$ and the convergence is smooth outside a singular set (which is a set of codimension at least 4) to a solution of a flow. We also prove that in the case of complex dimension 2, we can find a subsequence of times such that we have a convergence to a Kähler-Ricci soliton, away from finitely many isolated singularities.
\end{abstract}

\section{Introduction}

Let $M$ be a compact Kähler manifold of dimension $n$ with the Kähler metric $d s^{2}=g_{i \bar{j}} d z^{i} d \bar{z}^{j}$. The Ricci curvature of this metric is given by the formula

$$
R_{i \bar{j}}=\frac{-\partial^{2}}{\partial z^{i} \partial \bar{z}^{j}} \ln \operatorname{det}\left(g_{i \bar{j}}\right) .
$$

This implies that $\frac{\sqrt{-1}}{2 \pi} R_{i \bar{j}} d z^{i}$ andd $\bar{z}^{j}$ is closed and its cohomology class is equal to the first Chern class $c_{1}(M)$ of $M$. We will assume that $c_{1}(M)$ is positive and that it is represented by a Kähler form. We will consider the complex version of Hamilton's Ricci flow equation of the following type:

$$
\left(g_{i \bar{j}}\right)_{t}=g_{i \bar{j}}-R_{i \bar{j}}=\partial_{i} \bar{\partial}_{j} u,
$$

where $g_{i \bar{j}}(t)=g_{i \bar{j}}(0)+\partial_{i} \bar{\partial}_{j} \phi$ and $\frac{d}{d t} \phi=u$. In [2] H.D. Cao proved that a solution of (1) exists for all times $t \in[0, \infty)$. A natural question that one can ask is what happens to a flow when time approaches infinity. Under which conditions will it converge? How can we describe the objects that we get in a limit? In this paper we will give partial answers to these questions.

In section 3 we will consider a Kähler-Ricci flow (1) with uniformly bounded Ricci curvatures. Our goal is to prove the following theorem.

Theorem 1. Assume we are given a flow (1) in an arbitrary dimension. Assume that the Ricci curvatures are uniformly bounded, i.e. $\mid$ Ric $\mid \leq C$ for all $t$. Then for every sequence $t_{i} \rightarrow \infty$ there exists a subsequence such that $\left(M, g\left(t_{i}+t\right)\right) \rightarrow$ $(Y, \bar{g}(t))$ and the convergence is smooth outside a singular set $S$, which is at least of codimension four. Moreover, $\bar{g}(t)$ solves the Kähler-Ricci flow equation off the singular set.

Received by the editors May 13, 2004. 
In section 4 we will restrict ourselves to complex dimension 2 . We are interested in proving the convergence of a Kähler Ricci flow, under some reasonable assumptions, to Kähler Ricci solitons. It may happen that a Kähler Ricci soliton arising as a limit of the flow has singularities. A lot of progress has been made in studying the limits of a Kähler Ricci flow. It is especially important to know when we can expect to get Kähler Einstein metrics in a limit. Among first works on that topic is the work by Chen and Tian ([4], [5]) where they proved that if $M$ admits a Kähler Einstein metric with positive scalar curvature and if an initial metric has a nonnegative bisectional curvature that is positive at least at one point, then the Kähler Ricci flow converges exponentially fast to a Kähler Einstein metric with constant bisectional curvature. In [12], Phong and Sturm recently gave also some sufficient conditions for having a flow converge exponentially to a Kähler Einstein metric. In general, if a flow converges at least sequentially to Kähler Ricci solitons, it would be nice to see when we get a unique limit. A very important question of the uniqueness of Kähler Ricci solitons has been studied by Tian and Zhu in [14] and [15].

In this paper we also want to prove the following theorem.

Theorem 2. Let $g_{k \bar{j}}(t)=g_{k \bar{j}}-R_{k \bar{j}}$ be a Kähler-Ricci flow on a 2 dimensional complex, Kähler manifold with uniformly bounded Ricci curvatures. Then for every sequence $t_{i} \rightarrow \infty$ there exists a subsequence so that $\left(M, g\left(t_{i}+t\right)\right) \rightarrow$ $(Y, \bar{g}(t))$, where $Y$ is a smooth manifold outside finitely many isolated points and $\bar{g}(t)$ is a Kähler-Ricci soliton away those bad points.

\section{Background and notation}

First of all, let us recall the definitions of Ricci solitons.

Definition 3. A solution $g_{i \bar{j}}$ to equation (1) on $M$ is called Kähler-Ricci soliton if it moves along (1) under one-parameter family of automorphisms of $M$ generated by some holomorphic vector field.

This means that

$$
g_{i \bar{j}}-R_{i \bar{j}}=V_{i, \bar{j}}+V_{\bar{j}, i}
$$

for some holomorphic vector field $V=\left(V^{i}\right)$. In the case of limit solitons in Theorem 2, we will show that the vector fields come from the gradients of functions on $M$, i.e. that

$$
g_{i \bar{j}}-R_{i \bar{j}}=f_{, i \bar{j}},
$$

and $f_{, i j}=0$ for some real valued function $f$ on $M$. This condition is equivalent to a fact that $V=\nabla f$ is a holomorphic vector field.

Perelman's functional $\mathcal{W}$ for a flow (1) is

$$
\mathcal{W}(g, f, \tau)=(4 \pi \tau)^{-n} \int_{M} e^{-f}\left[2 \tau\left(R+|\nabla f|^{2}\right)+f-2 n\right] d V_{g}
$$


with a constraint that $(4 \pi \tau)^{-n} \int_{M} e^{-f} d V_{g}=1$. Perelman has proved some very interesting properties of flow (1). We will list them in the following theorem.

Theorem 4 (Perelman). If (1) is a flow on a complex, Kähler, closed manifold $M$, then

1. $C^{1, \alpha}$ norms of functions $u(t)$ are uniformly bounded along the flow,

2 . the scalar curvatures $R(t)$ and the diameters $\operatorname{diam}(M, g(t))$ are uniformly bounded along the flow,

3. a volume noncollapsing condition holds along the flow, i.e. there exists $C=C(g(0))$ such that $\operatorname{Vol}_{t}(B(p, r)) \geq C r^{n}$.

We will need a theorem proved by Cheeger, Colding and Tian ([15]) in our further discussion and we will state it below for a reader's convenience.

Theorem 5 (Cheeger, Colding, Tian). If $\left.\left\{M_{i}, g_{i}, p_{i}\right)\right\}$ converges to $(Y, d, y)$ in pointed Gromov-Hausdorff topology, if $|\operatorname{Ric}|_{M_{i}} \leq C$ and if $\operatorname{Vol}\left(B_{1}\left(p_{i}\right)\right) \geq C$ for all $i$, then the regular part $\mathcal{R}$ of $Y$ is a $C^{1, \alpha}$-Riemannian manifold and at points of $\mathcal{R}$, the convergence is $C^{1, \alpha}$. Moreover the codimension of the set of singular points (which is a closed set in $Y$ ) is at least 4 .

In the proof of Theorem 1 we will use Perelman's pseudolocality theorem ([11]).

Theorem 6 (Perelman). For every $\alpha>0$ there exist $\delta>0, \epsilon>0$ with the following property. Suppose we have a smooth solution to the Ricci flow and assume that at $t=0$ we have $R(x) \geq-r_{0}^{-2}$ and $\operatorname{Vol}(\partial \Omega)^{n} \geq(1-\delta) c_{n} \operatorname{Vol}(\Omega)^{n-1}$ for any $x, \Omega \subset B\left(x_{0}, r_{0}\right)$, where $c_{n}$ is the euclidean isoperimetric constant. Then, $|\operatorname{Rm}|(x, t) \leq \alpha t^{-1}+\left(\epsilon r_{0}\right)^{-2}$ whenever $0<t \leq\left(\epsilon r_{0}\right)^{2}$ and $\operatorname{dist}_{t}\left(x, x_{0}\right)<\epsilon r_{0}$.

Perelman proved this theorem for a case of unnormalized Ricci flow, but it can be easily modified for the case of a normalized Kähler-Ricci flow.

\section{Kähler-Ricci flow with uniformly bounded Ricci curvatures}

In this section we will consider a flow (1), with uniformly bounded Ricci curvatures. For any sequence $t_{i} \rightarrow \infty$, if $g_{i}(t)=g\left(t_{i}+t\right)$, the metrics $g_{i}(t)$ are uniformly equivalent to metrics $g_{i}(s)$ for $s, t$ belonging to an interval of finite length. Moreover, the following proposition (in [7]) applies to metrics $g_{i}(t)$.

Proposition 7 (D. Glickenstein). Let $\left\{\left(M_{i}, g_{i}(t), p_{i}\right)\right\}_{i=1}^{\infty}$, where $t \in[0, T]$, be a sequence of pointed Riemannian manifolds of dimension $n$ which is continuous in the $t$ variable in the following way: for each $\delta>0$ there exists $\eta>0$ such that if $t_{0}, t_{1} \in[0, T]$ satisfies $\left|t_{0}-t_{1}\right|<\eta$ then

$$
(1+\delta)^{-1} g_{i}\left(t_{0}\right) \leq g_{i}\left(t_{1}\right) \leq(1+\delta) g_{i}\left(t_{0}\right),
$$

for all $i>0$, and such that $\operatorname{Ric}\left(g_{i}(t)\right) \geq c g_{i}(t)$, where $c$ does not depend on $t$ or $i$. Then there is a subsequence $\left\{\left(M_{i}, g_{i}(t), p_{i}\right)\right\}_{i=1}^{\infty}$ and a 1-parameter family of complete pointed metric spaces $(X(t), d(t), x)$ such that for each $t \in[0, T]$ 
the subsequence converges to $(X(t), d(t), x)$ in the pointed Gromov-Hausdorff topology.

$\left(M_{i}, g_{i}(t)\right)$ and $\left(M_{i}, g_{i}(0)\right)$ are homeomorphic by Lipschitz homeomorphisms, and in [7] it has been showed be showed that $X(t)$ is homeomorphic to $X(0)$. If $t_{i}$ is any sequence such that $t_{i} \rightarrow \infty$, Proposition 7 applies to $\left(M, g\left(t_{i}+t\right)\right)$ for all $i$ and all $t$ belonging to a time interval of finite length.

For the moment we will restrict ourselves to the case of Kähler manifolds of complex dimension 2, and later we will show how it can be generalized to an arbitrary dimension. In the case of complex dimension 2, for every sequence $t_{i} \rightarrow \infty$ there is a subsequence $\left\{\left(M, g\left(t_{i}+t\right)\right)\right\}$ converging to a compact orbifold $(Y, \bar{g}(t))$ with isolated singularities. This is due to the fact that $L^{2}$ norm of the curvature operator in the Kähler case can be uniformly bounded in terms of the first and the second Chern class of a manifold and its Kähler class. Combining Proposition 7 and Theorem 5 gives that $(Y, \bar{g}(t))$ is an 1 parameter family of orbifolds (it is even a Lipschitz family for $t$ belonging to an interval of finite length), such that a regular part of $(Y, \bar{g}(t))$ is $C^{1, \alpha}$ manifold and the convergence $\left(M, g\left(t_{i}+t\right)\right) \rightarrow(Y, \bar{g}(t))$ takes place in $C^{1, \alpha}$ topology, away from the set of singular points (which is common for all orbifolds $(Y, \bar{g}(t))$ ). In the case of higher dimensions, again by Proposition 7 and Theorem 5 we will have that $\left\{\left(M, g\left(t_{i}+t\right)\right)\right\}$ converge to $(Y, \bar{g}(t))$ with a singular set $S \subset Y$ of codimension at least 4. $\mathcal{R}=Y \backslash S$ is an open $C^{1, \alpha}$ manifold and the convergence on $\mathcal{R}$ is in $C^{1, \alpha}$ norm. We will show later that the set $S$ is common for $(Y, \bar{g}(t))$ for all $t$. The main tools in the proof of Theorem 1 will be Theorem 6 and Theorem A.1.5 of Cheeger and Colding that can be found in the appendix of [6].

We will now prove Theorem 1.

Proof. If the curvature does not blow up, we are done. Therefore, assume that the curvature does blow up. Let $t_{i} \rightarrow \infty$ be such that $Q_{i}=|\operatorname{Rm}|\left(p_{i}, t_{i}\right) \geq$ $\max _{M \times\left[0, t_{i}\right]}|\operatorname{Rm}|(x, t)$ and $Q_{i} \rightarrow \infty$. We already know that since $|\operatorname{Ric}|(g(t)) \leq$ $C$, there exists a subsequence $\left(M, g\left(t_{i}+t\right)\right)$ converging to orbifolds $(Y, \bar{g}(t))$ in $C^{1, \alpha}$ norm off the set of singular points. Moreover, metrics $\bar{g}(t)$ are $C^{1, \alpha}$ off the singular set. We may assume that $\operatorname{Sing}(Y)=\{p\}$. Our goal is to show that we actually have $C^{\infty}$ convergence off the singular point $p$, due to the fact that our metrics are changing with the Kähler-Ricci flow.

Adopt the notation of [15]. In general, a point $y \in Y$ is called regular, if for some $k$, every tangent cone at $y$ is isometric to $\mathrm{R}^{k}$. Denote a set of those points by $\mathcal{R}_{k}$ and let $\mathcal{R}=\cup_{k} \mathcal{R}_{k}$. Because of the noncollapsing condition that we have because of Theorem 4, we have that $\mathcal{R}=\mathcal{R}_{n}$. Let $\mathcal{R}_{\epsilon}=\left\{y \mid d_{G H}\left(B_{1}\left(y_{\infty}\right), B_{1}(0)\right)<\right.$ $\epsilon$ for every tangent cone $\left.\left(Y_{y}, y_{\infty}\right)\right\}$, where $B_{1}(0)$ is a unit ball in $\mathrm{R}^{n}$. Let $\mathcal{R}_{\epsilon, r}$ be a set of all points $y \in Y$ such that there exists $x$ such that $(0, x) \in R^{4} \times\{x\}$ and for some $u>r$ and every $s \in(0, u] d_{G H}\left(B_{s}(y), B_{s}((0, x))\right)<\epsilon s . \mathcal{R}_{\epsilon}=\cup_{r} \mathcal{R}_{\epsilon, r}$.

Choose $\epsilon_{P}$ and $\delta_{P}$ as in Perelman's pseudolocality theorem. Choose $\epsilon^{\prime}>0$ such that $\delta_{P}>\epsilon^{\prime}$ and $\epsilon^{\prime} \leq \epsilon_{0}$, where $\epsilon_{0}$ is such that $\mathcal{R}=\mathcal{R}_{\epsilon}$ for all $\epsilon \leq \epsilon_{0}$ (the existence of such an $\epsilon_{0}$ is proved in section 7 of [6]. 
Fix a time $t=t_{0}$. Pick up any point $q \in Y \backslash\{p\}$. Then $q \in \cap_{\epsilon \leq \epsilon_{0}} \mathcal{R}_{\epsilon}$. Let $d=\operatorname{dist}_{\bar{g}\left(t_{0}\right)}(p, q)$.

Claim 8. There exist $\eta>0$ and a sequence $q_{i} \in M$ such that $q_{i} \rightarrow q$, while $\left(M, g\left(t_{i}+t\right)\right) \rightarrow(Y, \bar{g}(t))$ as $i \rightarrow \infty$ for all $t$.

Proof. Assume that $q \in K \subset Y \backslash\{p\}$, where $K$ is a compact set and let $r=$ $\operatorname{dist}(K, p)$. For every $t, g\left(t_{i}+t\right)$ uniformly converge to $\bar{g}(t)$ on $K$. Let $\phi: K \rightarrow K_{i}$ be diffeomorphisms as in a definition of convergence of $\left(M, g\left(t_{i}+t\right)\right)$ to $(Y, \bar{g}(t))$. Let $q_{i}\left(t_{0}\right) \in M$ be such that $\operatorname{dist}_{g\left(t_{i}+t_{0}\right)}\left(q_{i}\left(t_{0}\right), \phi_{i}(q)\right)<\epsilon$, for $i \geq i_{0}$. Since the Ricci curvatures of $g(t)$ are uniformly bounded, there exists $\eta>0$ so that $|t-s|<\eta$ implies that $\left|\operatorname{dist}_{g\left(t_{i}+s\right)}(x, y)-\operatorname{dist}_{g\left(t_{i}+t\right)}(x, y)\right|<\epsilon$, for all $x, y \in M$. Therefore,

$$
\operatorname{dist}_{g\left(t_{i}+t\right)}\left(q_{i}\left(t_{0}\right), \phi_{i}(q)\right) \leq \operatorname{dist}_{g\left(t_{i}+t_{0}\right)}\left(q_{i}\left(t_{0}\right), \phi_{i}(q)\right)+\epsilon<2 \epsilon,
$$

for $i \geq i_{0}$ and for all $t \in[-\eta, \eta]$. Notice that $\eta$ does not depend either on $t_{0}$ or $q$, but it depends on $K$, i.e. on its distance from $p$. Therefore, if we continue this process infinitely many times, considering $t_{0}+\eta$ instead of $t_{0}$, etc. we get that the sequence $\left\{q_{i}\right\}$ will work for all times $t \geq 0$.

Lemma 9. For any regular point $q \in \mathcal{R}$ there exists $i_{0}, \eta$ and $r>0$ such that for all $B_{g\left(t_{i}+t\right)}\left(s, q^{\prime}\right) \subset B_{g\left(t_{i}+t\right)}\left(r, q_{i}(t)\right)$ we have $\operatorname{Vol}_{g\left(t_{i}+t\right)} B_{g\left(t_{i}+t\right)}\left(s, q^{\prime}\right) \geq\left(1-\epsilon^{\prime}\right) s^{n}$, for all $i \geq i_{0}$ and all $t \in\left[t_{0}-\eta, t_{0}+\eta\right]$, where $q_{i} \in M$ is a sequence of points converging to $q$, while $\left(M, g\left(t_{i}+t\right)\right) \rightarrow(Y, \bar{g}(t))$.

Proof. For $\epsilon^{\prime}$ find $r$ and $\delta$ as in Theorem A.1.5 (i) and (ii) in [6]. For this $\delta$ (that now plays the role of $\epsilon$ in Theorem $A .1 .5$ in [6]) find $\delta_{1}$ and $r_{1}$ (by part (iii) of the same theorem), such that $x \in(\mathcal{W R})_{8 \delta_{1}, r^{\prime}}$ implies that

$$
y \in \mathcal{R}_{\delta, s} \quad \forall y \in B_{r^{\prime}}(x) \forall s \leq(1-\delta) r^{\prime}-\operatorname{dist}_{0}(x, y), \quad r^{\prime} \leq r_{1},
$$

where a distance is measured in metric $\bar{g}\left(t_{0}\right)$. We may assume that $r_{1}<d$, because otherwise we can decrease $r_{1}$. Take any sequence $\delta_{i} \rightarrow 0$ as $i \rightarrow \infty$. We can choose a sequence $r_{i}$ such that $q \in \mathcal{R}_{\delta_{i}, r_{i}}$, since $q \in \mathcal{R}_{\delta_{i}}$. We claim that $q \in \mathcal{R}_{\delta_{1}, r}$, for some $r<r_{1}$. In order to prove that, we may assume $r_{i} \rightarrow 0$ (otherwise if $r_{i} \geq \kappa$ for all $i, d_{G H}\left(B_{l}(q), B_{l}(0)\right) \leq l \delta_{i} \rightarrow 0$ for all $l \leq \kappa$ and therefore we would have $\operatorname{Vol} B_{l}(q)=\operatorname{Vol} B_{l}(0)$ for all $l \leq \kappa$, and $q \in \mathcal{R}_{\delta_{1}, s}$ for some $s<r_{1}$, by Theorem A.1.5, part (i) in [6]. Therefore, there exist $\delta^{\prime \prime}<\delta_{1}$ and $r^{\prime \prime}<r_{1}$ such that $q \in \mathcal{R}_{\delta^{\prime \prime}, r^{\prime \prime}}$. This implies $q \in \mathcal{R}_{\delta_{1}, r^{\prime \prime}}$, since $\delta^{\prime \prime}<\delta_{1}$. This is true in metric $\bar{g}\left(t_{0}\right)$.

Claim 10. There exist $\eta>0$ and $i_{0}$ such that $q_{i} \in \mathcal{R}_{\delta_{1}, r^{\prime \prime}}$ for all metrics $g\left(t_{i}+t\right)$ for $i \geq i_{0}$ and $t \in\left[t_{0}-\eta, t_{+} \eta\right]$.

Proof. $q \in \mathcal{R}_{\delta_{1}, r^{\prime \prime}}$ and therefore,

$$
d_{G H}\left(B_{s}(x, 0), B_{s}\left(q, t_{0}\right)\right)<s \delta_{1}
$$


for some $s<r^{\prime \prime}$. We can substitute space $\{x\} \times \mathrm{R}^{4}$ by $\mathrm{R}^{4}$ only and therefore we can write just $B_{s}(0)$ instead of $B_{s}(x, 0)$. Since the Ricci curvatures of $g(t)$ are uniformly bounded, there exists $\eta$ such that $\left|t-t_{0}\right|<\eta$ implies that

$$
d_{G H}\left(B_{g\left(t_{i}+t\right)}\left(q_{i}, s\right), B_{g\left(t_{i}+t_{0}\right)}\left(q_{i}, s\right)\right)<\delta_{1} s .
$$

Since $g\left(t_{i}+t_{0}\right)$ converges to $\bar{g}\left(t_{0}\right)$ uniformly, away from a singular point $p$, there exists $i_{0}$ (depending on $\delta_{1} s$ and a compact set $K$ ) such that for $i \geq i_{0}$

$$
d_{G H}\left(B_{g\left(t_{i}+t_{0}\right)}\left(q_{i}, s\right), B_{\bar{g}\left(t_{0}\right)}(q, s)\right)<\delta_{1} s .
$$

Combining estimates (4), (5) and (6), together with an approximate triangle inequality for Gromov-Hausdorff distance we get

$$
d_{G H}\left(B_{g\left(t_{i}+t\right)}\left(q_{i}, s\right), B_{s}(0)\right)<4 \delta_{1} s,
$$

for all $i \geq i_{0}$ and all $t \in\left[t_{0}-\eta, t_{0}+\eta\right]$. This implies that $q_{i} \in \mathcal{W} \mathcal{R}_{8 \delta_{1}, r^{\prime \prime}}$, for all $i \geq i_{0}$ and all $t \in\left[t_{0}-\eta, t_{0}+\eta\right]$.

Combining Claim 10 and part (iii) of Theorem A.1.5 in [6], we get that $q^{\prime} \in$ $\mathcal{R}_{\delta, s}$, for all $q^{\prime} \in B_{g\left(t_{i}+t\right)}\left(q_{i}, r^{\prime \prime}\right), s \leq(1-\delta) r^{\prime \prime}-\operatorname{dist}_{t_{i}+t}\left(q_{i}, q^{\prime}\right)$, for all $i \geq i_{0}$ and $t \in\left[t_{0}-\eta, t_{0}+\eta\right]$. Part (ii) of Theorem $A .1 .5$ in [6] gives that

$$
\operatorname{Vol} B_{g\left(t_{i}+t\right)}\left(s, q^{\prime}\right) \geq\left(1-\epsilon^{\prime}\right) \operatorname{Vol} B_{s}(0),
$$

for all $q^{\prime} \in B_{g\left(t_{i}+t\right)}\left(r^{\prime \prime}, q\right)$ and $s \leq(1-\delta) r^{\prime \prime}-\operatorname{dist}_{g\left(t_{i}+t\right)}\left(q_{i}, q^{\prime}\right)$. By reducing $r^{\prime \prime}$ we get that there exists $r^{\prime \prime}$ such that the estimate (7) holds for all $q^{\prime} \in$ $B_{g\left(t_{i}+t\right)}\left(r^{\prime \prime}, q\right)$ and all $s$ such that $B_{g\left(t_{i}+t\right)}\left(s, q^{\prime}\right) \subset B_{g\left(t_{i}+t\right)}\left(r^{\prime \prime}, q\right)$, for $i \geq i_{0}$ and $t \in\left[t_{0}-\eta, t_{0}+\eta\right]$.

Choose $r, i_{0}$ and $\eta$ as in the claim above (for our regular point $q$ that we have fixed earlier). Reduce $r^{\prime \prime}$ if necessary, so that $\left(\epsilon^{\prime} r^{\prime \prime}\right)^{2}<\eta$. Since $1-\epsilon^{\prime}>$ $1-\delta_{P}$, and since for every ball $B_{g\left(t_{i}-\left(\epsilon^{\prime} r^{\prime \prime}\right)^{2} / 2\right)}\left(q^{\prime}, s\right) \subset B_{g\left(t_{i}-\left(\epsilon^{\prime} r^{\prime \prime}\right)^{2} / 2\right)}\left(q_{i}, r^{\prime \prime}\right)$, we have that $\operatorname{Vol}_{g\left(t_{i}-\left(\epsilon^{\prime} r^{\prime \prime}\right)^{2} / 2\right)} B_{s}\left(q^{\prime}\right) \geq\left(1-\delta_{P}\right) s^{n} c_{n}$, by Perelman's pseudolocality Theorem 6

$$
|\mathrm{Rm}|(x, t) \leq \frac{1}{\left(\epsilon^{\prime} r^{\prime \prime}\right)^{2}}+\left(\epsilon^{\prime} r^{\prime \prime}\right)^{2}
$$

for all $x \in B_{g(t)}\left(q_{i}, \epsilon^{\prime} r^{\prime \prime}\right)$ and for every $t \in\left[t_{i}-\left(\epsilon^{\prime} r^{\prime \prime}\right)^{2} / 2, t_{i}+\left(\epsilon^{\prime} r^{\prime \prime}\right)^{2} / 2\right]$. We have that $g_{i}(t)=g\left(t_{i}+t\right)$ is a sequence of Ricci flows with uniformly bounded curvatures for $t \in\left[-\left(\epsilon^{\prime} r^{\prime \prime}\right)^{2} / 2,\left(\epsilon^{\prime} r^{\prime \prime}\right)^{2} / 2\right]$ on balls $B_{g_{i}(t)}\left(q_{i}, \epsilon^{\prime} r^{\prime \prime}\right)$. This together with the volume noncollapsing condition and Hamilton's compactness theorem give that the convergence of the sequence of our metrics is smooth, and $\bar{g}(t)$ are smooth metrics on $B_{\bar{g}(t)}\left(q, \epsilon^{\prime} r^{\prime \prime}\right)$, for $t \in\left[0,\left(\epsilon^{\prime} r^{\prime \prime}\right)^{2} / 2\right]$. Repeating the procedure described above infinitely many times, to time intervals translated by $\left(\epsilon^{\prime} r^{\prime \prime}\right)^{2} / 2$ (considering $t_{0}+\left(\epsilon^{\prime} r^{\prime \prime}\right)^{2} / 2$ instead of $t_{0}$, etc.) and applying diagonalization method to a sequence of times $t_{i}$ (since for every step of length $\left(\epsilon^{\prime} r^{\prime \prime}\right)^{2} / 2$ we have to extract a subsequence of a subsequence), we get that $\bar{g}(t)$ are smooth metrics on $B\left(q, \epsilon^{\prime} r^{\prime \prime}\right)$ for all times $t \geq 0$ (we can take $t_{0}=0$ ) and that $g\left(t_{i}+t\right) \rightarrow \bar{g}(t)$ 
smoothly on $B_{\bar{g}(t)}\left(q, \frac{\epsilon^{\prime} r^{\prime \prime}}{2}\right)$ for all times $t \geq 0$. We will use the fact that the Ricci tensor is uniformly bounded to show that we can extend the previous result from a ball to any compact set $K \subset Y$. By a definition of convergence, that will mean $\left(M, g\left(t_{i}+t\right)\right) \rightarrow(Y, \bar{g}(t))$ smoothly, away from the set of singular points.

Take a compact set $K \subset Y \backslash S$, where $S$ is a set of singular points on $(Y, \bar{g}(t))$. It is the same set for all singular metrics $\bar{g}(t)$. Let $\phi_{i}: K \rightarrow K_{i}$ be a sequence of diffeomorphisms from a definition of convergence of metrics $g_{i}(0)$ to a metric $\bar{g}(0)$.

$|\operatorname{Ric}|(t) \leq C$ for all $t$ by the assumtion of the main theorem. We have proved that $\bar{g}(t)$ is 1-parameter family of metrics on $Y$. Moreover, $\bar{g}(t)$ satisfies the Kähler-Ricci flow equation away from the singular points.

Claim 11. There exist $\delta>0$, a subsequence $t_{i}$ and $C_{1}=C_{1}(K)$ such that $|\operatorname{Rm}|\left(g\left(t_{i}+t\right)\right) \leq C$ on $K_{i}$ for all $t \in\left[t_{0}, t_{0} \delta\right]$ and all $t_{0}$.

Proof. Fix $t_{0}$. For every $q \in Y$ we can choose $r_{q}>0, \eta_{q}$ and $i_{q}$ as in Lemma 9. Look at the collection of balls $B_{\bar{g}\left(t_{0}\right)}\left(q,\left(\epsilon^{\prime} r_{q}\right) / 4\right)$ covering $K$. Since $K$ is compact we can consider only finitely many of them covering $K$. Denote their centres by $q_{1}, q_{2}, \ldots q_{N}$. Since $\bar{g}(t)$ solves the equation (1) and since the Ricci curvatures of $\bar{g}(t)$ are uniformly bounded on $Y \backslash\{p\}$, there exists $A>0$ so that the balls $B_{\bar{g}(t)}\left(q_{i},\left(\epsilon^{\prime} r_{q_{i}}\right) / 2\right)$ cover $K$, for $t \in\left[t_{0}-A, t_{0}+A\right]$. Let $r_{1}=\min \left\{r_{q_{1}}, r_{q_{2}}, \ldots, r_{q_{N}}\right\}, \eta_{1}=\min \left\{\eta_{q_{1}}, \ldots, \eta_{q_{N}}\right\}$ and $i_{1}=\max \left\{i_{q_{1}}, \ldots i_{q_{N}}\right\}$. Then $|\operatorname{Rm}|\left(x, t+t_{i}\right) \leq \frac{1}{\left(\epsilon^{\prime} r_{1}\right)^{2}}+\left(\epsilon^{\prime} r_{1}\right)^{2}=C_{1}(K)$ for all $x \in B_{g\left(t_{i}+t\right)}\left(q_{i}^{j}, \epsilon^{\prime} r_{q_{j}}\right)$, all $i \geq i_{1}$ and all $t \in\left[0, \min \left\{\eta_{1},\left(\epsilon^{\prime} r_{1}\right)^{2} / 2\right\}\right]$, where $q_{i}^{j}$ are the sequences of points such that $B_{g_{\left(t_{i}+t\right)}}\left(q_{i}^{j}, r_{q_{j}}\right) \rightarrow B_{\bar{g}(t)}\left(q_{j}, r_{q_{j}}\right)$ while $\left(M, g\left(t_{i}+t\right)\right) \rightarrow(Y, \bar{g}(t))$. Let $\left.\delta=\min \left\{\eta_{1},\left(\epsilon^{\prime} r_{1}\right)^{2} / 2, A\right\}\right]$.

The balls $B_{\bar{g}(t)}\left(q_{j},\left(\epsilon^{\prime} r_{q_{j}}\right) / 2\right)$ for $1 \leq j \leq N$ cover $K$. A definition of convergence gives that there exists $i_{0} \geq i_{1}$ so that $B_{g\left(t_{i}\right)}\left(q_{i}^{j}, \frac{2 \epsilon^{\prime} r_{q_{j}}}{3}\right)$ cover $K_{i}$ for all $i \geq i_{0}$. We can assume that $\delta$ is small enough so that

$$
B_{g\left(t_{i}\right)}\left(q_{i}^{j},\left(r_{q_{j}}-a\right) \epsilon^{\prime}\right) \subset B_{g\left(t_{i}+s\right)}\left(q_{i}^{j}, r_{q_{j}} \epsilon^{\prime} / 2\right),
$$

for $a<\frac{r_{1}}{3}$ so that $r_{q_{j}}-a>r_{q_{j}}-\frac{r_{1}}{3}>\frac{2 r_{q_{j}}}{3}$ and therefore $B_{g\left(t_{i}\right)}\left(q_{i}^{j},\left(\epsilon^{\prime}\left(2 r_{q_{j}}\right) / 3\right) \subset\right.$ $B_{g\left(t_{i}\right)}\left(q_{i}^{j},\left(r_{q_{i}^{j}}-a\right) \epsilon^{\prime}\right)$. Since the balls $B_{g\left(t_{i}\right)}\left(q_{i}^{j},\left(2 r_{q_{j}} \epsilon^{\prime}\right) / 3\right)$ cover $K_{i}$, so do balls $B_{g\left(t_{i}+s\right)}\left(q_{i}^{j}, r_{q_{j}}\right)$ for all $i \geq i_{0}$ and all $s \in\left[t_{0}-\delta, t_{0}+\delta\right]$. Therefore, $|\operatorname{Rm}|\left(x, t_{i}+s\right) \leq$ $C_{1}$ for all $i \geq i_{1}$ and all $x \in K_{i}$ and all $s \in\left[t_{0}-\delta, t_{0}+\delta\right]$. Therefore we actually can extract a subsequence $t_{i}$ such that the pullbacks of metrics $g\left(t_{i}+s\right)$ converge to a solution of the Kähler-Ricci flow uniformly on $K \times\left[t_{0}-\delta, t_{0}+\delta\right]$.

Applying the method from the previous claim to a sequence $t_{i}+\delta$ instead of a sequence $t_{i}$ we can find a subsequence such that $g\left(t_{i}+s\right) \rightarrow \bar{g}(t)$ smoothly and uniformly on $K \times\left[t_{0}, t_{0}+2 \delta\right]$, since our choice of $\delta$ does not depend on an initial time, but on a chosen compact set $K \subset Y$ and a uniform bound on the Ricci tensor. Repeating this infinitely many times and diagonalizing the sequence $t_{i}$, we get a sequence $t_{i}$ such that $g\left(t_{i}+s\right) \rightarrow \bar{g}(t)$ smoothly converge on all 
compact subsets of $K \times[0, \infty)$. We can choose a countable sequence of compact sets $L_{k}$ exhausting $Y \backslash S$. We can find a subsequence of $t_{i}$ for each $L_{k}$, so that the above that we have proved for any compact set $K \subset Y \backslash S$, applies to $L_{k}$ as well. By a diagonalization procedure applied to $t_{i}$ we can get a subsequence so that $\left(M, g\left(t_{i}+t\right) \rightarrow(Y, \bar{g}(t))\right.$ for all $t \geq 0$, where $\bar{g}(t)$ is a solution to the Kähler-Ricci flow away from the set of singular points. The convergence is in the sense that for every compact set $K \subset Y \backslash S$ there exist diffeomorphisms $\phi_{i}: K \rightarrow K_{i}$, where $K_{i} \subset M$ are compact and $\phi_{i}^{*} g\left(t_{i}+t\right) \rightarrow \bar{g}(t)$, uniformly and smoothly on all compact subsets of $K \times[0, \infty)$.

In the proof of Theorem 1 we assumed that the complex dimension was 2 . The proof above generalizes to an arbitrary dimension easily. Since the Ricci tensor is uniformly bounded along the flow, for every sequence $t_{i} \rightarrow \infty$ there exists a subsequence so that $\left(M, g\left(t_{i}+t\right)\right) \rightarrow(Y(t), \bar{g}(t))$ and the convergence is smooth outside a set $S(t)$ of codimension at least 4 . As above, it easily follows that $Y=Y(t)$ for all $t$. We should only check that $S(t)=S(s)$ for any $s, t \in[0, \infty)$.

Lemma 12. $S(s)=S(t)$ for any $s, t \in[0, \infty)$.

Proof. It is enough to prove: $\exists a>0$ such that for $|s-t|<a S(s)=S(t)$.

Choose $\epsilon>0$ such that $\mathcal{R}_{\epsilon}(s)=\mathcal{R}(s)$ and $\mathcal{R}_{\epsilon}(t)=\mathcal{R}(t)$, for $|s-t|<a$, where we will choose $a$ later. Assume there exists $q \in S(t) \backslash S(s)$. That implies $q \in \mathcal{R}(s)$. For $\epsilon>0$ choose $\epsilon^{\prime}=\epsilon^{\prime}(\epsilon, n)>0$ and $r^{\prime}=r^{\prime}(\epsilon, n)$ so that Theorem A.1.5 in [6] holds. Then the following claim holds for $q$.

Claim 13. There exist $i_{0}$ and $r<r^{\prime}$ such that for all $B_{g\left(t_{i}+s\right)}\left(q^{\prime}, u\right) \subset$ $B_{g\left(t_{i}+s\right)}\left(q_{i}, r\right)$ we have $\operatorname{Vol}_{g\left(t_{i}+s\right)} B_{g\left(t_{i}+s\right)}\left(q^{\prime}, u\right) \geq c_{n}\left(1-\epsilon^{\prime} / 2\right) u^{n}$, for all $i \geq i_{0}$, where $q_{i} \in M$ is a sequence of points converging to $q$, while $\left(M, g\left(t_{i}+s\right)\right) \rightarrow$ $(Y, \bar{g}(s))$.

The proof of this claim is the same as the proof of Lemma 9.

Since the Ricci tensors are uniformly bounded along the flow, we have a good control on the volumes and the sizes of balls in metrics at different times, when the considered time interval is sufficiently small. Similarly as in [8] we can find sufficiently small $a>0$ such that $|s-t|<a$, for any $u<r$ implies that

$$
\begin{gathered}
\operatorname{Vol}_{g\left(t_{i}+t\right)} B_{g\left(t_{i}+t\right)}(q, u) \geq \sqrt{\left(1-\frac{\epsilon^{\prime}}{2}\right)} \operatorname{Vol}_{g\left(t_{i}+s\right)} B_{g\left(t_{i}+s\right)}(q, u) \geq\left(1-\frac{\epsilon^{\prime}}{2}\right), \\
B_{g\left(t_{i}+s\right)}(q, u \tilde{r}) \subset B_{g\left(t_{i}+t\right)}(q, u),
\end{gathered}
$$

where $\tilde{r}=\frac{1}{\left.1+\left(e^{2 C|s-t|}-1\right)^{\frac{1}{2}}\right)}$ and we can choose $a$ small enough, so that $\tilde{r}^{n}>$ $\sqrt{1-\frac{\epsilon^{\prime}}{2}}$. Finally, since $\operatorname{Vol}_{g\left(t_{i}+s\right)} B_{g\left(t_{i}+s\right)}(q, u) \geq c_{n}\left(1-\frac{\epsilon^{\prime}}{2}\right) u^{n}$, we get that

$$
\operatorname{Vol}_{g\left(t_{i}+t\right)} B_{g\left(t_{i}+t\right)}(q, u) \geq\left(1-\epsilon^{\prime} / 2\right)^{2} u^{n} c_{n} \geq\left(1-\epsilon^{\prime}\right) c_{n} u^{n}
$$

i.e. $q \in \mathcal{R}_{\epsilon, r / 2} \subset \mathcal{R}_{\epsilon}=\mathcal{R}(t)$. This means that $q$ can not be in $S(t)$ and we get a contradiction. We can repeat the procedure above infinitely many times to get 
that $S(t)=S(s)$ for all $s, t \in\left[t_{0}, t_{0}+a\right]$ and all $t_{0} \geq 0$, i.e. $S(t)=S$ for all $t \geq 0$.

Having Lemma 12 we can repeat the proof of Theorem 1 for complex dimension 2 , to get that theorem is actually true for all dimensions.

\section{Kähler-Ricci soliton as a limit}

In this section we want to prove Theorem 2. By Theorem 1 we can find a subsequence $t_{i}$ so that $\left(M, g\left(t_{i}+t\right)\right) \rightarrow(Y, \bar{g}(t))$, where $Y$ is a smooth manifold outside a closed set $S$ of codimension 4 . Since we are in complex dimension 2, a set of codimension four is a set of points and since it is a closed subset of a compact set $Y$ ( $Y$ is compact, since it comes from a limit of compact manifolds with uniformly bounded diameters), $S$ is just a set of finitely many singular points.

Claim 14. There is a finite $\lim _{t \rightarrow \infty} \mu(g(t), 1 / 2)$.

Proof.

$$
\mu(g(t), 1 / 2) \leq(2 \pi)^{-n} \int_{M} e^{-u}\left(|\nabla u|^{2}+R+u-2 n\right) d V_{g(t)} \leq C,
$$

for a uniform constant $C$, since scalar curvature and $C^{1}$ norm of Ricci potentials $u(t)$ are uniformly bounded along the Kähler Ricci flow by results of Perelman. He also proved that $\mu(g(t), 1 / 2)$ is a monotone quantity along the flow and therefore there is a finite $\lim _{t \rightarrow \infty} \mu(g(t), 1 / 2)$ as claimed.

By results from [3] we have that

$$
\begin{aligned}
\mu\left(g\left(t_{i}+A\right), 1 / 2\right)-\mu\left(g\left(t_{i}\right), 1 / 2\right) & \\
= & \int_{0}^{A} \frac{d}{d t} \mu\left(g\left(t_{i}+t\right), 1 / 2\right) d t \\
= & (2 \pi)^{-n} \int_{0}^{A} \int_{M}\left|R_{j \bar{k}}\left(t_{i}+t\right)+\nabla_{j} \nabla_{\bar{k}} f\left(t_{i}+t\right)-g_{j \bar{k}}\right|^{2} d V_{g\left(t_{i}+t\right)} d t+ \\
& 2(2 \pi)^{-n} \int_{0}^{A} \int_{M}\left|\nabla_{i} \nabla_{j} f\left(t_{i}+t\right)\right|^{2} d V_{t_{i}+t} d t
\end{aligned}
$$

where $f\left(t_{i}+t\right)$ is a minimizer for $\mathcal{W}$ with respect to metric $g(t)$. Since $\mu\left(g\left(t_{i}+A\right), 1 / 2\right)-\mu\left(g\left(t_{i}\right), 1 / 2\right) \rightarrow 0$ as $i \rightarrow \infty$, we have that

$$
\lim _{i \rightarrow \infty} R_{j \bar{k}}\left(t_{i}+t\right)+\nabla_{j} \nabla_{\bar{k}} f\left(t_{i}+t\right)-g_{j \bar{k}}\left(t_{i}+t\right)=0
$$

and

$$
\lim _{i \rightarrow \infty} \nabla_{j} \nabla_{k} f\left(t_{i}+t\right)=0
$$

for almost all $t \in[0, A]$ and almost all $x \in M$. Assume we are having only one singular point, say $p$. Since the convergence is smooth away from it, by taking a sequence of radii $r_{k} \rightarrow 0$ as $k \rightarrow \infty$, since the geometries of $g\left(t_{i}+t\right)$ are uniformly bounded in $i$, depending on $r_{k}$ only, on $M \backslash B_{t_{i}}\left(p_{i}, r_{k}\right)$, for each $k$ 
we can extract a subsequence such that $g\left(t_{i}+t\right) \rightarrow \bar{g}(t)$ uniformly on compact subsets of $Y \backslash B_{\bar{g}(0)}\left(p, r_{k}\right)$ for $t \in[0, A]$ and therefore,

$$
\operatorname{Ric}(\bar{g}(t))+\nabla \bar{\nabla} \tilde{f}(t)-\bar{g}(t)=0,
$$

and $\nabla \nabla \tilde{f}=0$ on $Y \backslash B_{\bar{g}(0)}\left(p, r_{k}\right)$, for $t \in[0, A]$, where $\tilde{f}(t)$ is a limit of $f\left(t_{i}+t\right)$. By letting $k \rightarrow \infty$ and finding a diagonal sequence, we can find a subsequence $t_{i}$ so that $g\left(t_{i}+t\right) \rightarrow \bar{g}(t)$ uniformly on compact subsets of $Y \backslash\{p\}$, for $t \in[0, A]$ and $\bar{g}(t)$ satisfies the soliton equation (8) away from sigular points. By taking $A$ bigger and bigger, again by diagonalizing a sequence $t_{i}$, by uniqueness of a limit we get a subsequence $t_{i}$ so that $g\left(t_{i}+t\right) \rightarrow \bar{g}(t)$ away from sigular points, for

all $t \in[0, \infty)$ and $\bar{g}(t)$ satisfies (8) with a soliton potential function $\tilde{f}$ satisfying $\tilde{f}_{i j}=\tilde{f}_{\bar{j} \bar{k}}=0$, which tells us that $\nabla \tilde{f}$ defines a holomorphic vector field, that is, $\bar{g}(t)$ satisfies a Kähler Ricci soliton equation away from singular points.

\section{Acknowledgements}

The author would like to thank her advisor Gang Tian for constant help, support and many useful discussions.

\section{References}

[1] M. Anderson, Convergence and rigidity of manifolds under Ricci curvature bounds, Invent. Math. 102 (1990) 429-445.

[2] H.D. Cao, Deformation of Kähler metrics on compact Kähler manifolds, Invent. Math. 81 (1985) 359-372.

[3] H.D. Cao, R.Hamilton, T.Ilmanen, Gaussian densities and stability for some Ricci solitons, http://www.math.ethz.ch/ilmanen/papers/gaussrep.pdf.

[4] X.X. Chen, X.X., G.Tian, Ricci flow on Kähler Einstein surfaces, Invent. Math. 147 (2002) 487-544.

[5] , Ricci flow on Kähler manifolds, C.R.Acad.Sci. Paris Sér. I Math. 332 (2001) 245-248; also arXiv.math.DG/0108179.

[6] J. Cheeger, T.H. Colding, On the structure spaces with Ricci curvature bounded below, I. J. Differential Geom. 45 (1997) 406-480.

[7] D. Glickenstein, Precompactness of solutions to the Ricci flow in the absence of injectivity radius estimates, preprint arXiv:math.DG/0211191 v2.

[8] N. Sesum, Cuvature tensor under the Ricci flow. Preprint arXiv:math.DG/0311397.

[9] _ Limiting behaviour of the Ricci flow. Preprint arXiv:math.DG/0402194.

[10] N. Sesum, G. Tian, Bounding the scalar curvature and the diameter under the Kähler Ricciflow, in preparation.

[11] G. Perelman, The entropy formula for the Ricci flow and its geometric applications. Preprint arXiv:math.DG/0211159.

[12] D.H.Phong, J. Sturm, On stability and convergence of the Kähler Ricci flow, arXiv:math.DG/0412185.

[13] J. Cheeger, T.H. Colding, G.Tian, On the singularities of spaces with bounded Ricci curvature, Geom. Funct. Anal. 12 (2002) 873-914.

[14] G.Tian, X.H.Zhu, Uniqueness of Kähler Ricci solitons; Acta Math. 184 (2000) 271-305.

[15] _ A new holomorphic invariant and uniqueness of Kähler Ricci solitons, Comm.Math.Helv. 77 (2002) 297-325.

Department of Mathematics, New York University, New York, NY 1027

E-mail address: natasas@CIMS.nyu.edu 